\title{
Stereoselective Addition of
}

\section{Dimethyl Thiophosphite to Imines}

Pakamas Tongcharoensirikul, ${ }^{\dagger}$ Alirica I. Suarez, ${ }^{\dagger \ddagger}$ Troy Voelker, ${ }^{\S}$ and Charles M. Thompson ${ }^{\dagger{ }^{\dagger *}}$

${ }^{\dagger}$ Department of Biomedical and Pharmaceutical Sciences and ${ }^{\S}$ Department of Chemistry, The University of Montana, Missoula, MT 59812,

${ }^{\ddagger}$ Facultad de Farmacia, Universidad Central de Venezuela, Caracas, Venezuela

\section{Supporting Information}

\section{Contents:}

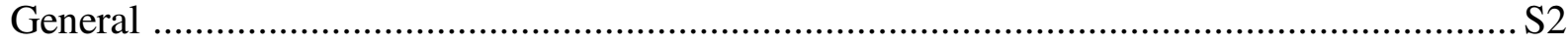

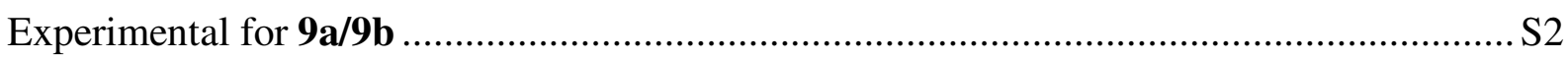

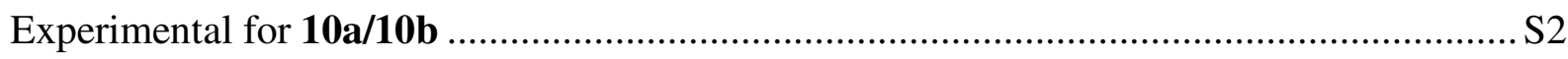

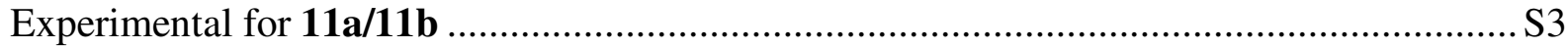

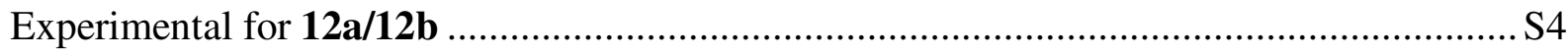

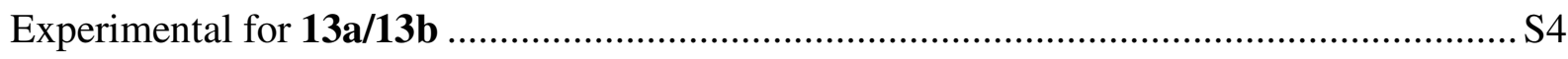

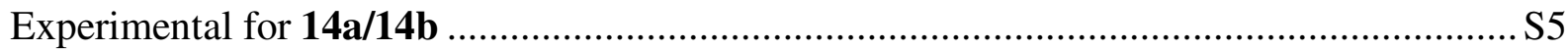

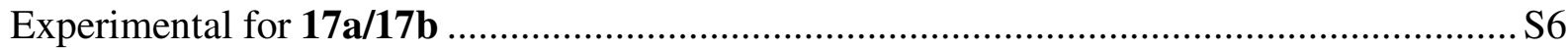

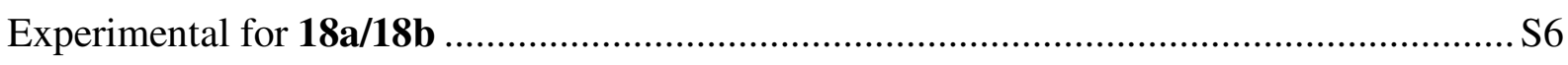

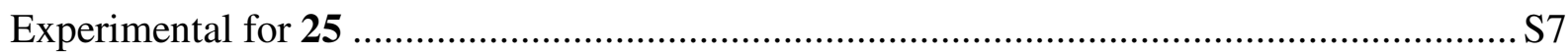


General Methods. Unless otherwise noted, reactions were run in flame-dried apparatus under argon atmosphere. Anhydrous solvents and reagents (amino acids, phenylglycinol, $\alpha$-methylbenzylamine, Pearlmann's catalyst) were used without further purification unless indicated. Molecular sieves ( $3 \AA$ A $)$ were flame dried prior to use. Analytical thin-layer chromatography (TLC) was performed using aluminum-backed, $0.2 \mathrm{~mm}$ silica gel plates. Flash chromatography was performed using silica gel 60, 230-240 mesh. Phosphorus- and nitrogen-containing compounds were detected using UV and/or stained with ninhydrin or 2,6-dibromoquinone-chlorimide. ${ }^{1} \mathrm{H}$ NMR (400 MHz), ${ }^{13} \mathrm{C}$ NMR (100 MHz), and ${ }^{31} \mathrm{P}$ NMR $(171 \mathrm{MHz})$ spectra were recorded on a $400 \mathrm{MHz}$ spectrometer in $\mathrm{CDCl}_{3}$ unless indicated. Chemical shifts $(\delta)$ are reported using $\mathrm{CHCl}_{3}\left(7.26 \mathrm{ppm}\right.$ for $\left.{ }^{1} \mathrm{H}\right), \mathrm{CDCl}_{3}\left(77 \mathrm{ppm}\right.$ for $\left.{ }^{13} \mathrm{C}\right)$ or $\mathrm{H}_{3} \mathrm{PO}_{4}\left(0\right.$ ppm for $\left.{ }^{31} \mathrm{P}\right)$ as references. High resolution mass spectra (HRMS) were obtained using electrospray ionization (ESI)/time-of-flight mass spectrometry (LC-TOF). Mass spectrometer samples were introduced using a HPLC fitted with a C-18 reversed phase column $(2.1 \mathrm{~mm}$ i.d., $5 \mathrm{~cm})$. Warning: Dimethyl thiophosphite generates a stench when distilled or upon contact with water.

\section{O,O-Dimethyl $(R S)$-\{[(1R)-2-hydroxy-1-phenylethyl]amino\}(phenyl)methyl-phosphonothioate 9a/9b:}

Benzaldehyde $(0.38 \mathrm{~mL}, 0.40 \mathrm{~g}, 3.74 \mathrm{mmol})$ was added to the solution of $(\mathrm{R})-(+)-2$-phenylglycinol $(0.51 \mathrm{~g}, 3.72$ $\mathrm{mmol})$ in toluene $(10 \mathrm{~mL})$ containing molecular sieves $(3 \AA, 0.5 \mathrm{~g})$. The reaction mixture was stirred at $\mathrm{rt}$ for $2 \mathrm{~h}$ or until an aliquot showed completion by the presence of the imino proton at $\delta 8.51$. The reaction was filtered through Celite and dimethyl thiophosphite $5(0.31 \mathrm{~mL}, 0.46 \mathrm{~g}, 3.69 \mathrm{mmol})$ was added. The reaction mixture was stirred at 0 ${ }^{\circ} \mathrm{C}$ for $12 \mathrm{~h}$, concentrated to an oil and subjected to column chromatography (1:1, hexanes:Et $\left.{ }_{2} \mathrm{O}\right)$. A major diastereomer 9a $(0.5776 \mathrm{~g}, 1.6 \mathrm{mmol}, 44.2 \%)$, minor diastereomer $9 \mathbf{b}(0.14 \mathrm{~g}, 0.40 \mathrm{mmol}, 10.7 \%)$ and mixture of diastereomers $(0.27 \mathrm{~g}, 0.77 \mathrm{mmol}, 20.6 \%)$ were obtained. All NMR spectroscopic data are identical to $\mathbf{1 7} \mathbf{a} / \mathbf{1 7} \mathbf{b}$. Elemental analyses performed on $\mathbf{1 7 a / 1 7 b}$.

$$
\text { 9a (major): }[\alpha]_{\mathrm{D}}^{23}=-34.63\left(\mathrm{c}=0.58, \mathrm{CHCl}_{3}\right) \text {. }
$$




\section{Methyl (2S)-2-\{[(RS)-(dimethoxyphosphorothioyl)(phenyl)methyl]amino\}-propanoate 10a/10b: To a} suspension of (S)-(-)-alanine methyl ester hydrochloride (1.78 g, $12.7 \mathrm{mmol})$ in THF (25 $\mathrm{mL})$ at $\mathrm{rt}$ was added TEA (1.77 mL, $1.29 \mathrm{~g}, 12.7 \mathrm{mmol})$. The mixture was stirred at $\mathrm{rt}$ for $30 \mathrm{~min}$ whereupon the mixture was filtered, and evaporated to yield crude alanine methyl ester as a colorless oil $(0.629 \mathrm{~g}, 6.10 \mathrm{mmol}, 48 \%)$. Toluene (10 mL), benzaldehyde $(0.62 \mathrm{~mL}, 0.65 \mathrm{~g}, 6.10 \mathrm{mmol})$ and molecular sieves $(3 \AA$ ) $)$ were added to the alanine methyl ester and the reaction mixture was stirred at rt. After $2 \mathrm{~h}$, the mixture was filtered through Celite and an aliquot showed complete imine formation (singlet @ $\delta 8.42 \mathrm{ppm})$. Dimethyl thiophosphite $5(0.51 \mathrm{~mL}, 0.77 \mathrm{~g}, 6.10 \mathrm{mmol}$ ) was added at $0{ }^{\circ} \mathrm{C}$ and the reaction mixture was stirred at $0{ }^{\circ} \mathrm{C}$ for $48 \mathrm{~h} . \mathrm{A}^{31} \mathrm{P}$ NMR of the crude showed two signals at $\delta$ 98.8/97.8 with the integration of 53:47, respectively. The reaction mixture was chromatographed on silica gel (hexanes: $\left.\mathrm{Et}_{2} \mathrm{O}, 95: 5\right)$ to afford 10a/10b as a yellowish oil $\left(0.33 \mathrm{~g}, 1.04 \mathrm{mmol}, 17 \%\right.$ ): TLC $R_{f}=0.08$ (hexanes:Et ${ }_{2} \mathrm{O}$, 9:1); ${ }^{1} \mathrm{H} \delta 7.45-7.29(\mathrm{~m}, 5 \mathrm{H}), 4.24\left(\mathrm{~d}, J_{P C H}=16 \mathrm{~Hz}, 0.5 \mathrm{H}\right), 4.20\left(\mathrm{~d}, J_{P C H}=19.6 \mathrm{~Hz}, 0.5 \mathrm{H}\right), 3.77\left(\mathrm{~d}, J_{P O C H 3}=13.2\right.$ $\mathrm{Hz}, 1.5 \mathrm{H}), 3.71\left(\mathrm{~d}, J_{\text {РОСН }}=12.8 \mathrm{~Hz}, 1.5 \mathrm{H}\right), 3.68(\mathrm{~s}, 1.5 \mathrm{H}), 3.56\left(\mathrm{~d}, J_{\text {РОСН } 3}=13.6 \mathrm{~Hz}, 1.5 \mathrm{H}\right), 3.54(\mathrm{~s}, 1.5 \mathrm{H}), 3.41$ $\left(\mathrm{d}, J_{\text {POCH } 3}=12.8 \mathrm{~Hz}, 1.5 \mathrm{H}\right), 3.47(\mathrm{q}, J=7.1 \mathrm{~Hz}, 0.5 \mathrm{H}), 3.21(\mathrm{q}, J=7.1 \mathrm{~Hz}, 0.5 \mathrm{H}), 1.30(\mathrm{~d}, J=7.1 \mathrm{~Hz}, 1.5 \mathrm{H}), 1.28$ $(\mathrm{d}, J=7.1 \mathrm{~Hz}, 1.5 \mathrm{H}) ;{ }^{13} \mathrm{C} \delta 175.2,174.7,134.9,134.5,134.4,129.1,129.0,128.8,128.7,128.4,128.3,127.2$, 127.1, $64.4\left(\mathrm{~d}, J_{\text {POСH } 3}=118.3 \mathrm{~Hz}\right), 63.9\left(\mathrm{~d} J_{\text {РОСН3}}=124.4 \mathrm{~Hz}\right), 54.6\left(\mathrm{~d}, J_{P O C H 3}=6.1 \mathrm{~Hz}\right), 54.2\left(\mathrm{~d}, J_{\text {POCH3 }}=6.1 \mathrm{~Hz}\right)$, $54.1\left(\mathrm{~d}, J_{\text {РОСН3 }}=6.1 \mathrm{~Hz}\right), 53.7,53.5,53.9\left(\mathrm{~d}, J_{\text {РОСН } 3}=9.1 \mathrm{~Hz}\right), 51.9,51.8,19.4,17.9 ;{ }^{31} \mathrm{P} \delta 98.3,97.2 ; \mathrm{HRMS}$ calcd for $\mathrm{M}+\mathrm{Na}^{+}$of $\mathrm{C}_{13} \mathrm{H}_{20} \mathrm{NO}_{4} \mathrm{PSNa} 340.0748$, found 340.0747 .

\section{Methyl (2S)-2-\{[(1RS)-1-(dimethoxyphosphorothioyl)ethyl]amino\}-3-hydroxypropanoate 11a/11b: To a} suspension of serine methyl ester hydrochloride $(1.73 \mathrm{~g}, 11.1 \mathrm{mmol})$ in THF $(30 \mathrm{~mL})$ was added TEA $(1.4 \mathrm{~mL}, 1.02$ $\mathrm{g}, 10.1 \mathrm{mmol}$ ) at rt. The reaction mixture was stirred at $\mathrm{rt}$ for $0.5 \mathrm{~h}$, filtered through Celite and the solvent removed to yield serine methyl ester as a clear liquid $(0.69 \mathrm{~g}, 5.8 \mathrm{mmol}, 57.5 \%)$. Toluene $(10 \mathrm{~mL})$ was added followed by acetaldehyde $(0.28 \mathrm{~mL}, 0.22 \mathrm{~g}, 5.0 \mathrm{mmol})$ and molecular sieves ( $3 \AA$, $0.5 \mathrm{~g})$. The reaction mixture was stirred at $\mathrm{rt}$ for $2 \mathrm{~h}$ and filtered through Celite. Dimethyl thiophosphite $5(0.3 \mathrm{~mL}, 0.45 \mathrm{~g}, 3.57 \mathrm{mmol})$ was added at $0{ }^{\circ} \mathrm{C}$ and the reaction mixture maintained at $0{ }^{\circ} \mathrm{C}$ for $48 \mathrm{~h}$. The ${ }^{31} \mathrm{P}$ NMR of the crude showed two signals at $\delta 102.6$ and 101.9 integrating to 55:45. The solvent was removed and the crude mixture chromatographed on silica gel 
$\left(\mathrm{CH}_{2} \mathrm{Cl}_{2}\right.$ :EtOAc, 9:1) to afford the diastereomers as a yellowish liquid $(0.32,1.17 \mathrm{mmol}, 32.7 \%)$. HRMS calcd for $\mathrm{M}+\mathrm{Na}^{+}$of $\mathrm{C}_{8} \mathrm{H}_{18} \mathrm{NO}_{5} \mathrm{PS} 294.0541$, found 294.0527.

11a (major): $\mathrm{TLC} R_{f}=0.3\left(\mathrm{CH}_{2} \mathrm{Cl}_{2}: \operatorname{EtOAc}, 4: 1\right) ;{ }^{1} \mathrm{H} \mathrm{NMR} \delta 3.75\left(\mathrm{~d}, J_{\text {POCH }}=13.2 \mathrm{~Hz}, 3 \mathrm{H}\right), 3.75(\mathrm{~s}, 3 \mathrm{H})$, $3.74\left(\mathrm{~d}, J_{\text {РОСН } 3}=12.0 \mathrm{~Hz}, 3 \mathrm{H}\right), 3.74\left(\mathrm{dd}, J_{\text {РСнNНСH }}=11.5,5.9 \mathrm{~Hz}, 2 \mathrm{H}\right), 3.63(\mathrm{dd}, J=11.5,5.9 \mathrm{~Hz}, 1 \mathrm{H}), 3.16(\mathrm{qd}$, $\left.J_{P C H}=15.0,7.1 \mathrm{~Hz}, 1 \mathrm{H}\right), 2.75(\mathrm{br} \mathrm{s}, 2 \mathrm{H}), 1.33\left(\mathrm{dd}, J_{\text {PCHCH3 }}=18.8,7.1 \mathrm{~Hz}, 3 \mathrm{H}\right) ;{ }^{13} \mathrm{C} \mathrm{NMR} \delta 172.5,62.4,60.4,54.1$ $\left(\mathrm{d}, J_{P C}=151.8 \mathrm{~Hz}\right), 53.7\left(\mathrm{~d}, J_{\text {POCH }}=9.1 \mathrm{~Hz}\right), 53.3\left(\mathrm{~d}, J_{\text {POCH }}=9.1 \mathrm{~Hz}\right), 52.3,15.6 ;{ }^{31} \mathrm{P} \mathrm{NMR} \delta 102.6$.

$11 \mathrm{~b}$ (minor): TLC $R_{f}=0.26\left(\mathrm{CH}_{2} \mathrm{Cl}_{2}:\right.$ EtOAc, $\left.4: 1\right) ;{ }^{1} \mathrm{H}$ NMR $\delta 3.77\left(\mathrm{dd}, J_{P C H N H C H 2}=11.5,7.3 \mathrm{~Hz}, 2 \mathrm{H}\right)$, $3.76\left(\mathrm{~d}, J_{\text {РОСН3 }}=13.2 \mathrm{~Hz}, 6 \mathrm{H}\right), 3.76(\mathrm{~s}, 3 \mathrm{H}), 3.67(\mathrm{dd}, J=11.5,7.3 \mathrm{~Hz}, 1 \mathrm{H}), 3.10\left(\mathrm{dq}, J_{P C H}=15.2,7.0 \mathrm{~Hz}, 1 \mathrm{H}\right)$, 2.67 (br s, $2 \mathrm{H}), 1.36\left(\mathrm{dd}, J_{\text {РСНСН3}}=19.2,7.0 \mathrm{~Hz}, 3 \mathrm{H}\right) ;{ }^{13} \mathrm{C}$ NMR $\delta 173.0,62.8,60.2,55.4\left(\mathrm{~d}, J_{C-P}=130.5 \mathrm{~Hz}\right), 53.5$ $\left(\mathrm{d}, J_{\text {POCH3 }}=9.1 \mathrm{~Hz}\right), 53.4\left(\mathrm{~d}, J_{\text {POCH3 }}=9.1 \mathrm{~Hz}\right), 52.2,15.7 ;{ }^{31} \mathrm{P}$ NMR $\delta 101.9$.

\section{Methyl (2S)-2-\{[(1RS)-1-(dimethoxyphosphorothioyl)-2-methylpropyl]amino\}-3-hydroxypropanoate 12a/12b:}

To a suspension of (S)-L-serine methyl ester hydrochloride $(0.547 \mathrm{~g}, 3.52 \mathrm{mmol})$ in THF (15 mL) was added TEA $(0.49 \mathrm{~mL}, 0.356 \mathrm{~g}, 3.52 \mathrm{mmol})$. The reaction was stirred at $0{ }^{\circ} \mathrm{C}$ for $30 \mathrm{~min}$, filtered and the solvent removed under reduced pressure to give L-serine methyl ester as yellow oil $(0.256 \mathrm{~g}, 2.15 \mathrm{mmol})$. Isobutyraldehyde $(0.19 \mathrm{~mL}$, $0.155 \mathrm{~g}, 2.15 \mathrm{mmol})$ was added to a solution of L-serine methyl ester in $\mathrm{CH}_{2} \mathrm{Cl}_{2}(5 \mathrm{~mL})$ in the presence of molecular sieves ( $3 \AA ̊ .,(0.5 \mathrm{~g})$. The reaction mixture was stirred at $\mathrm{rt}$ for $2 \mathrm{~h}$, filtered through Celite and dimethyl thiophosphite $5(0.18 \mathrm{~mL}, 0.27 \mathrm{~g}, 2.14 \mathrm{mmol})$ was added. The reaction mixture was stirred at $0{ }^{\circ} \mathrm{C}$ for $12 \mathrm{~h}$ and the solvent removed to give the crude product $(0.429 \mathrm{~g})$ as a yellowish oil. The crude product was chromatographed on silica gel $\left(\mathrm{CH}_{2} \mathrm{Cl}_{2}\right.$ :hexanes, $\left.7: 3\right)$ to yield an inseparable mixture of diastereomers $(0.095 \mathrm{~g} 0.32 \mathrm{mmol}, 15 \%)$ : Anal. Calcd for: $\mathrm{C}_{10} \mathrm{H}_{22} \mathrm{NO}_{5} \mathrm{PS}, \mathrm{C}, 40.13 ; \mathrm{H}, 7.41 ; \mathrm{N}, 4.68$. Found: $\mathrm{C}, 40.03 ; \mathrm{H}, 7.31 ; \mathrm{N}, 4.56 ;{ }^{1} \mathrm{H}$ NMR $\delta 3.79-3.55$ (m, $\left.12 \mathrm{H}\right)$, $2.94\left(\mathrm{dd}, J_{P C H}=12.8,3.2 \mathrm{~Hz}, 0.66 \mathrm{H}\right), 2.80\left(\mathrm{dd}, J_{P C H}=11.2,2.4 \mathrm{~Hz}, 0.34 \mathrm{H}\right), 2.35-2.27(\mathrm{~m}, 1 \mathrm{H}), 0.99(\mathrm{~d}, J=6.4 \mathrm{~Hz}$, $4 \mathrm{H}), 0.95(\mathrm{~d}, J=6.4 \mathrm{~Hz}, 2 \mathrm{H}) ;{ }^{13} \mathrm{C} \mathrm{NMR} \delta 172.7,172.5,66.4,64.3\left(J_{\mathrm{CP}}=176 \mathrm{~Hz}\right), 64.2\left(J_{\mathrm{CP}}=182 \mathrm{~Hz}\right), 64.1,63.0$, $53.9\left(J_{\mathrm{POCH} 3}=6.1 \mathrm{~Hz}\right), 53.4\left(J_{\mathrm{POCH} 3}=6.0 \mathrm{~Hz}\right), 53.0,52.4\left(J_{\mathrm{PCH} 3}=6.1 \mathrm{~Hz}\right), 52.2\left(J_{\mathrm{POCH} 3}=6.0 \mathrm{~Hz}\right), 29.5(\mathrm{~d}, J=9.1$ Hz), $28.8(\mathrm{~d}, J=9 \mathrm{~Hz}), 21.6,21.4,21.3,21.1,17.1,16.7 ;{ }^{31} \mathrm{P}$ NMR $\delta 102.9,101.4$. 


\section{Methyl (2S)-2-\{[(RS)-(dimethoxyphosphorothioyl)(phenyl)methyl]amino\}-3-hydroxypropanoate 13a/13b: To} a suspension of (S)-L-serine methyl ester hydrochloride $(0.54 \mathrm{~g}, 3.47 \mathrm{mmol})$ in THF $(15 \mathrm{~mL})$ was added TEA $(0.48$ $\mathrm{mL}, 0.35 \mathrm{~g}, 3.44 \mathrm{mmol})$. The reaction was stirred at $0{ }^{\circ} \mathrm{C}$ for $30 \mathrm{~min}$ and filtered rapidly under vacuum. The solvent was evaporated to dryness to give L-serine methyl ester as an off-yellow oil ( $0.34 \mathrm{~g}, 2.82 \mathrm{mmol})$. To a solution of Lserine, methyl ester in $\mathrm{CH}_{2} \mathrm{Cl}_{2}(5 \mathrm{~mL})$ was added benzaldehyde $(0.27 \mathrm{~mL}, 0.28 \mathrm{~g}, 2.66 \mathrm{mmol})$ and molecular sieves (3 Å, $0.5 \mathrm{~g}$ ). The reaction mixture was stirred at $\mathrm{rt}$ for $2 \mathrm{~h}$ and filtered through Celite. Dimethyl thiophosphite 5 $(0.24 \mathrm{~mL}, 0.36 \mathrm{~g}, 2.85 \mathrm{mmol})$ was added and the reaction mixture was stirred at $0{ }^{\circ} \mathrm{C}$ for $12 \mathrm{~h}$. The solvent was evaporated to dryness to give crude product $(0.688 \mathrm{~g})$ as yellowish oil. The crude was purified by column chromatography $\left(\mathrm{CH}_{2} \mathrm{Cl}_{2}\right.$ :hexanes, 7:3). The products were isolated as a major diastereomer 13a $(0.41 \mathrm{~g}, 1.23 \mathrm{~mol}$, $46.2 \%)$, a minor diastereomer $\mathbf{1 3 b}(0.05 \mathrm{~g}, 0.016 \mathrm{mmol}, 6.2 \%)$ and a mixture of diastereomers $\mathbf{1 3 a} / \mathbf{1 3 b}(0.09 \mathrm{~g}, 0.27$ mmol, 10.0\%). Anal. Calcd for : $\mathrm{C}_{13} \mathrm{H}_{20} \mathrm{NO}_{5} \mathrm{PS}: \mathrm{C}, 46.84 ; \mathrm{H}, 6.05 ; \mathrm{N}, 4.20$; Found: C, 46.49; H, 5.96; N, 4.21.

13a (major): TLC $R_{f}=0.30\left(\mathrm{CH}_{2} \mathrm{Cl}_{2}: \mathrm{EtOAC}, 9: 1\right) ;[\alpha]^{23}{ }_{\mathrm{D}}=+4.0\left(\mathrm{c}=1.0, \mathrm{CHCl}_{3}\right) ;{ }^{1} \mathrm{H} \mathrm{NMR} \delta$ 7.39-7.26 $(\mathrm{m}, 5 \mathrm{H}), 4.16\left(\mathrm{~d}, J_{C P H}=20.4 \mathrm{~Hz}, 1 \mathrm{H}\right), 3.81-3.55(\mathrm{~m}, 2 \mathrm{H}), 3.75\left(\mathrm{~d}, J_{P O C H 3}=13.2 \mathrm{~Hz}, 3 \mathrm{H}\right), 3.60(\mathrm{~s}, 3 \mathrm{H}), 3.45-3.32$ $(\mathrm{m}, 1 \mathrm{H}), 3.38\left(\mathrm{~d}, J_{\text {РОСH3 }}=13.6 \mathrm{~Hz}, 3 \mathrm{H}\right), 2.91(\mathrm{br} \mathrm{s}, 1 \mathrm{H}) ;{ }^{13} \mathrm{C} \mathrm{NMR} \delta 172.4,134.7,128.5,128.5,128.3,63.7\left(\mathrm{~d}, J_{P C}\right.$ $=128.1 \mathrm{~Hz}), 61.6,60.2,54.3\left(\mathrm{~d}, J_{\text {РОСН } 3}=9.1 \mathrm{~Hz}\right), 53.7\left(\mathrm{~d}, J_{\text {РОСН } 3}=6.1 \mathrm{~Hz}\right), 52.2 ;{ }^{31} \mathrm{P} \mathrm{NMR} \delta 97.8$.

13b (minor): TLC $R_{f}=0.24\left(\mathrm{CH}_{2} \mathrm{Cl}_{2}:\right.$ EtOAc, $\left.9: 1\right) ;{ }^{1} \mathrm{H}$ NMR $\delta$ 7.42-7.26 (m, 5H), $4.29\left(\mathrm{~d}, J_{P C H}=15.6 \mathrm{~Hz}\right.$, $1 \mathrm{H}), 3.74-3.64(\mathrm{~m}, 2 \mathrm{H}), 3.69\left(\mathrm{~d}, J_{\text {POСH } 3}=13.2 \mathrm{~Hz}, 3 \mathrm{H}\right), 3.70(\mathrm{~s}, 3 \mathrm{H}), 3.53\left(\mathrm{~d}, J_{\text {POCH } 3}=13.2 \mathrm{~Hz}, 3 \mathrm{H}\right), 3.35-3.30(\mathrm{~m}$, $1 \mathrm{H}) ;{ }^{13} \mathrm{C}$ NMR $\delta 172.6,134.2,129.1,129.1,128.4,128.3,64.2\left(\mathrm{~d}, J_{P C}=119.1 \mathrm{~Hz}\right), 63.1,60.0,54.3\left(\mathrm{~d}, J_{\text {POСН }}=6.1\right.$ $\mathrm{Hz}), 53.7\left(\mathrm{~d}, J_{\text {POCH3 }}=6.1 \mathrm{~Hz}\right), 52.1 ;{ }^{31} \mathrm{P} \mathrm{NMR} \delta 97.2$.

\footnotetext{
Methyl (2S, 3R)-2-\{[(RS)-(dimethoxyphosphorothioyl)(phenyl)methyl]amino\}-3-hydroxybutanoate 14a/14b: Benzaldehyde $(0.79 \mathrm{~mL}, 0.828 \mathrm{~g}, 7.8 \mathrm{mmol})$ was added to a solution of L-threonine methyl ester $(1.039 \mathrm{~g}, 7.8 \mathrm{mmol})$ in $\mathrm{CH}_{2} \mathrm{Cl}_{2}(5 \mathrm{~mL})$ in the presence of $\mathrm{K}_{2} \mathrm{CO}_{3}$. The reaction mixture was stirred at $\mathrm{rt}$ for $2 \mathrm{~h}$ and filtered through Celite. The solvent was evaporated to dryness to give crude imine $(1.481 \mathrm{~g})$ as a yellowish oil. Dimethyl thiophosphite $5(0.56 \mathrm{~mL}, 0.84 \mathrm{~g}, 6.66 \mathrm{mmol})$ was added directly to the imine resulting in a viscous solution. ${ }^{31} \mathrm{P}$ NMR of an aliquot showed diastereomer formation in a ratio of 62:38. The crude was purified by column
} 
chromatography $\left(\mathrm{CH}_{2} \mathrm{Cl}_{2}\right.$ :hexanes, $\left.7: 3\right)$ to give a mixture of diastereomers $(0.777 \mathrm{~g}, 2.24 \mathrm{mmol}, 33.6 \%)$ as a yellow oil; HRMS calcd for $\mathrm{M}+\mathrm{H}^{+}$of $\mathrm{C}_{14} \mathrm{H}_{23} \mathrm{NO}_{5} \mathrm{PS} 348.1034$, found 348.1046 .

14a (minor): ${ }^{1} \mathrm{H}$ NMR $\delta$ 7.40-7.20 (m, 5H), $4.07\left(\mathrm{~d}, 1 \mathrm{H}, J_{P C H}=14.8 \mathrm{~Hz}\right), 3.86-3.79(\mathrm{~m}, 1 \mathrm{H}), 3.75(\mathrm{~d}, 3 \mathrm{H}$, $\left.J_{\text {POСH } 3}=13.6 \mathrm{~Hz}\right), 3.37(\mathrm{~s}, 3 \mathrm{H}), 3.33\left(\mathrm{~d}, 3 \mathrm{H}, J_{\text {POСH } 3}=12.8 \mathrm{~Hz}\right), 2.95(\mathrm{~d}, 1 \mathrm{H}, J=6.4 \mathrm{~Hz}), 1.15(\mathrm{~d}, 3 \mathrm{H}, J=6.4 \mathrm{~Hz})$;

${ }^{13} \mathrm{C}$ NMR $\delta 173.20,134.9,133.8,129.2,128.7,128.6,128.4,68.2,66.7,65.9\left(\mathrm{~d}, J_{C P}=127.50 \mathrm{~Hz}\right), 54.2\left(\mathrm{~d}, J_{\text {РОСн3 }}=\right.$ 9.1 Hz), $53.7\left(\mathrm{~d}, J_{\text {РОСН3 }}=6.1 \mathrm{~Hz}\right) 51.8,19.1 .{ }^{31} \mathrm{P} \mathrm{NMR} \delta 97.2$.

14b (major): TLC $R_{f}=0.50\left(\mathrm{CH}_{2} \mathrm{Cl}_{2}: \operatorname{EtOAc}, 4: 1\right) ;{ }^{1} \mathrm{H}$ NMR $\delta 7.40-7.20(\mathrm{~m}, 5 \mathrm{H}), 4.11\left(\mathrm{~d}, J_{P C H}=14 \mathrm{~Hz}, 1\right.$ $\mathrm{H}), 3.75-3.70(\mathrm{~m}, 1 \mathrm{H}), 3.67(\mathrm{~s}, 3 \mathrm{H}), 3.64\left(\mathrm{~d}, J_{\text {PCH } 3}=13.6 \mathrm{~Hz}, 3 \mathrm{H}\right), 3.52\left(\mathrm{~d}, J_{\text {POCH }}=13.6 \mathrm{~Hz}, 3 \mathrm{H}\right), 2.99(\mathrm{~d}, 1 \mathrm{H}, J$ $=6.4 \mathrm{~Hz}) 1.13(\mathrm{~d}, J=6.4 \mathrm{~Hz}, 3 \mathrm{H}) ;{ }^{13} \mathrm{C} \mathrm{NMR} \delta 173.0,134.9,133.8,129.2,129.1,128.7,128.4,67.9,66.6,64.5(\mathrm{~d}$, $\left.J_{C P}=118.4 \mathrm{~Hz}\right), 54.3\left(\mathrm{~d}, J_{\mathrm{POCH} 3}=6.1 \mathrm{~Hz}\right), 53.6\left(\mathrm{~d}, J_{\mathrm{POCH} 3}=6.1 \mathrm{~Hz}\right) 51.9,19.3 .{ }^{31} \mathrm{P} \mathrm{NMR} \delta 96.8$.

O,O-Dimethyl (RS)-\{[(1S)-2-hydroxy-1-phenylethyl]amino\}(phenyl)methyl-phosphonothioate 17a/17b: The identical procedure to $\mathbf{9 a} / \mathbf{9 b}$ was conducted except using $(S)$-phenylglycinol. A major diastereomer $\mathbf{1 7 b}(0.59 \mathrm{~g}$, $1.68 \mathrm{mmol}, 44.9 \%)$, minor diastereomer $17 \mathbf{a}(0.08 \mathrm{~g}, 0.23 \mathrm{mmol}, 6.2 \%)$ and mixture of diastereomers $(0.16 \mathrm{~g}, 0.46$ mmol, $12.3 \%$ ) were obtained following chromatography. Anal. Calcd for $\mathrm{C}_{17} \mathrm{H}_{22} \mathrm{NO}_{3} \mathrm{PS}: \mathrm{C}, 58.11 ; \mathrm{H}, 6.31 ; \mathrm{N}, 3.99$. Found: C, 57.90; H, 6.42; N, 3.89 .

17a (minor): $\operatorname{TLC} R_{f}=0.32\left(1: 4\right.$, hexanes: $\left.\mathrm{Et}_{2} \mathrm{O}\right) ;{ }^{1} \mathrm{H}$ NMR $\delta 7.36-7.21(\mathrm{~m}, 10 \mathrm{H}), 3.97\left(\mathrm{~d}, J_{P C H}=18.8 \mathrm{~Hz}\right.$, $1 \mathrm{H}), 3.74\left(\mathrm{~d}, J_{\mathrm{POCH}}=12.8 \mathrm{~Hz}, 3 \mathrm{H}\right) 3.68-3.58(\mathrm{~m}, 3 \mathrm{H}), 3.45\left(\mathrm{~d}, J_{\text {POСH }}=12.8 \mathrm{~Hz}, 3 \mathrm{H}\right), 2.28(\mathrm{br} \mathrm{s}, 1 \mathrm{H}) ;{ }^{13} \mathrm{C} \mathrm{NMR} \delta$ $138.7,134.4,129.0,128.9,128.7,128.4,128.3,127.9,127.6,67.0,62.3\left(\mathrm{~d}, J_{P C}=122.0 \mathrm{~Hz}\right), 61.5,61.3,54.1(\mathrm{~d}$, $\left.J_{\text {POCH3 }}=6.1 \mathrm{~Hz}\right) 53.3\left(\mathrm{~d}, J_{\mathrm{POCH} 3}=6.1 \mathrm{~Hz}\right) ;{ }^{31} \mathrm{P} \mathrm{NMR} \delta 98.1$.

17b (major): $\operatorname{TLC} R_{f}=0.27\left(1: 4\right.$, hexanes: $\left.\mathrm{Et}_{2} \mathrm{O}\right) ;[\alpha]^{23}{ }_{\mathrm{D}}=+35.32\left(\mathrm{c}=1.09, \mathrm{CHCl}_{3}\right) ;{ }^{1} \mathrm{H} \mathrm{NMR} \delta$ 7.33-7.21 $(\mathrm{m}, 10 \mathrm{H}), 4.20\left(\mathrm{~d}, J_{P C H}=17.2 \mathrm{~Hz}, 1 \mathrm{H}\right), 4.01-3.95(\mathrm{~m}, 1 \mathrm{H}), 3.78\left(\mathrm{~d}, J_{\text {РОСH }}=15.6 \mathrm{~Hz}, 3 \mathrm{H}\right), 3.79-3.73(\mathrm{~m}, 1 \mathrm{H}), 3.32$ $\left(\mathrm{d}, J_{\text {РОСн3 }}=12.8 \mathrm{~Hz}, 3 \mathrm{H}\right), 2.64(\mathrm{br} \mathrm{s}, 1 \mathrm{H}) ;{ }^{13} \mathrm{C} \mathrm{NMR} \delta 140.0,135.7,128.5,128.4,128.3,128.1,127.7,127.4,66.0$, $62.9\left(\mathrm{~d}, J_{P C}=116.0 \mathrm{~Hz}\right), 62.5,62.4,54.5\left(\mathrm{~d}, J_{\text {РОСН } 3}=6.1 \mathrm{~Hz}\right), 53.8\left(\mathrm{~d}, J_{\text {РОСН } 3}=9.1 \mathrm{~Hz}\right) ;{ }^{31} \mathrm{P}$ NMR $\delta 100.5$. 
O,O-Dimethyl (RS)-phenyl $\{[(1 S)$-1-phenylethyl]amino\}methylphosphonothioate 18a/18b: Benzaldehyde $(0.63$ $\mathrm{mL}, 0.66 \mathrm{~g}, 6.2 \mathrm{mmol})$ was added to the solution of $(R)-(+)-\alpha-$ methylbenzylamine $(0.80 \mathrm{~mL}, 0.75 \mathrm{~g}, 6.2 \mathrm{mmol})$ in toluene $(10 \mathrm{~mL})$ in the presence of molecular sieves $(3 \AA, 0.5 \mathrm{~g})$. The reaction mixture was stirred at $\mathrm{rt}$ for $2 \mathrm{~h}$ and was filtered through a pad of Celite under Ar. Dimethyl thiophosphite $5(0.52 \mathrm{~mL}, 0.78 \mathrm{~g}, 6.20 \mathrm{mmol})$ was added to the solution of imine at $0{ }^{\circ} \mathrm{C}$ and the reaction mixture was stirred at $0{ }^{\circ} \mathrm{C}$ for $12 \mathrm{~h}\left({ }^{31} \mathrm{P}\right.$ NMR showed signals at $\delta$ 101.6 and 98.9 integrating to $76: 24$ ). The crude was chromatographed on silica gel (hexanes:Et ${ }_{2} \mathrm{O}, 98: 2$ ) to afford the major diastereomer $\mathbf{1 8 b}(1.32 \mathrm{~g}, 3.95 \mathrm{mmol}, 63.8 \%)$ : $\mathrm{TLC} R_{f}=0.44$ (hexanes: $\left.\mathrm{Et}_{2} \mathrm{O}=8: 2\right) ;{ }^{1} \mathrm{H}$ NMR $\delta$ 7.37-7.21 $(\mathrm{m}, 10 \mathrm{H}), 4.24\left(\mathrm{~d}, J_{P C H}=19.2 \mathrm{~Hz}, 1 \mathrm{H}\right), 3.93(\mathrm{q}, J=6.4 \mathrm{~Hz}, 1 \mathrm{H}), 3.84\left(\mathrm{~d}, J_{\text {POCH } 3}=12.8 \mathrm{~Hz}, 3 \mathrm{H}\right), 3.36\left(\mathrm{~d}, J_{P O C H}=\right.$ $12.8 \mathrm{~Hz}, 3 \mathrm{H}), 2.45$ (br s, 1H), $1.36(\mathrm{~d}, J=6.4 \mathrm{~Hz}, 3 \mathrm{H}) ;{ }^{13} \mathrm{C}$ NMR $\delta 145.0,135.9,128.4,128.4,128.3,128.1,127.0$, 126.7, $62.6\left(\mathrm{~d}, J_{C P}=115.3 \mathrm{~Hz}\right), 55.2\left(\mathrm{~d}, J_{\text {POСH } 3}=9.1 \mathrm{~Hz}\right), 54.1\left(\mathrm{~d}, J_{\text {POСH } 3}=6.1 \mathrm{~Hz}\right), 53.7,23.0 ;{ }^{31} \mathrm{P} \mathrm{NMR} \delta 101.6$; HRMS calcd for $\mathrm{M}+\mathrm{H}^{+}$of $\mathrm{C}_{17} \mathrm{H}_{22} \mathrm{NO}_{2} \mathrm{PS}$ calcd for 336.1187, found 336.1194 .

(S)-Phosphonophenylglycine 25: ${ }^{24}$ Hydrolysis of the enantiomer 24 using the identical procedure to the preparation of 22 affords (S)-phosphonophenylglycine 25 (65\%). The product 25 obtained exhibited the same spectral data as obtained for 22 except $[\alpha]^{23}=-16.20(\mathrm{c}=0.60,1 \mathrm{~N} \mathrm{NaOH})$. 